\title{
Geometric Fixed Point Theorems on Transversal Spaces
}

\author{
Milan R. TASKOVIĆ* \\ Dedicated to professor Ranko Bojanić on his $80^{\text {th }}$ birthday.
}

ABstract. This paper presents new fixed point theorems on lower and upper transversal spaces. The following main result is proved that if $T$ is a self-map on an orbitally DS-complete transversal lower space $(X, \rho)$, if there exists an upper semicontinuous function $G: X \rightarrow \mathbb{R}$ such that

$$
\rho[x, T x] \geq G(T x)-G(x) \geq 0
$$

for every $x \in X$ and if $G\left(T^{n} a\right) \rightarrow+\infty$ as $n \rightarrow \infty$ for some $a \in X$, then $T$ has a fixed point in $X$. For the lower transversal spaces are essential the mappings $T: X \rightarrow X$ which are unbounded variation, i.e., if there exists a function $A: X \times X \rightarrow \mathbb{R}_{+}^{0}$ such that

$$
\sum_{n=0}^{\infty} A\left(T^{n} x, T^{n+1} x\right)=+\infty
$$

for arbitrary $x \in X$. On the other hand, for upper transversal spaces are essential the mappings $T: X \rightarrow X$ which are bounded variation

\section{INTRODUCTION AND HISTORY}

The possibility of defining such notions as limit and continuity in an arbitrary set is an idea which undoubtedly was first put forward by M. Fréchet in 1904, and developed by him in his famous thesis in 1906 .

The simplest and most fruitful method which be proposed for such definitions was the introduction of the notion of distance.

But the greatest merit of Fréchet lies in the emphasis he put on three notions which were to play a fundamental part in all later developments of Functional Analysis: compactness, completeness, and separability.

In this sense, the notion of order, and the notion of new completeness, have each led to a fixed point statement. We now obtain geometric results of fixed

1991 Mathematics Subject Classification. Primary: 54E15, 47H10, 05A15. Secondary: 54E35, $54 \mathrm{H} 25$.

Key words and phrases. Fixed point theorems, lower and upper transversal spaces, orbitally DS-complete and CS-complete spaces, functions of unbounded variation, function of bounded variation, geometric fixed point theorems.

${ }^{*}$ Research supported by Science Fund of Serbia under Grant 1457. 
points based on an interpley of these two notions as new notations in transversal upper and lower spaces.

In connection with this, first, in Tasković [16] we introduced the concept of transversal (upper and lower) spaces as a natural extension of Fréchet's, Kurepa's and Menger's spaces.

Let $X$ be a nonempty set. The function $\rho: X \times X \rightarrow \mathbb{R}_{+}^{0}:=[0,+\infty)$ is called an upper transverse on $X$ (or upper transversal) iff: $\rho[x, y]=\rho[y, x], \rho[x, y]=0$ if and only if $x=y$, and if there is function $\psi:\left(\mathbb{R}_{+}^{0}\right)^{2} \rightarrow \mathbb{R}_{+}^{0}$ such that

$$
\rho[x, y] \leq \max \{\rho[x, z], \rho[z, y], \psi(\rho[x, z], \rho[z, y])\}
$$

for all $x, y, z \in X$. An upper transversal space is a set $X$ together with a given upper transverse on $X$. The function $\psi$ in (As) is called upper bisection function.

On the other hand, the function $\rho: X \times X \rightarrow[0,+\infty]:=\mathbb{R}_{+}^{0} \cup\{+\infty\}$ is called a lower transverse on $X$ (or lower transversal) iff: $\rho[x, y]=\rho[y, x], \rho[x, y]=+\infty$ if and only if $x=y$, and if there is a lower bisection function $d:[0,+\infty]^{2} \rightarrow[0,+\infty]$ such that

$$
\rho[x, y] \geq \min \{\rho[x, z], \rho[z, y], d(\rho[x, z], \rho[z, y])\}
$$

for all $x, y, z \in X$. A lower transversal space is a set $X$ together with a given lower transverse on $X$. The function $d$ in $(\mathrm{Am})$ is called lower bisection function.

In recent years a great number of papers have presented results in the fixed point theory on miscellaneous spaces.

In this paper we begin with intensive development of the fixed point theory on transversal spaces, special, with development on lower transversal spaces.

\section{FiXED POINTS ON LOWER TRANSVERSAL SPACES}

Let $(X, \rho)$ be a lower transversal space and $T: X \rightarrow X$. We shall introduce the concept of DS-convergence in a space $X$; i.e., a lower transversal space $X$ satisfies the condition of DS-convergence (or $X$ is DS-complete) iff: $\left\{x_{n}\right\}_{n \in \mathbb{N}}$ is an arbitrary sequence in $X$ and $\sum_{i=1}^{\infty} \rho\left[x_{i}, x_{i+1}\right]=+\infty$ implies that $\left\{x_{n}\right\}_{n \in \mathbb{N}}$ has a convergent subsequence in $X$.

In connection with this, a lower transversal space $X$ satisfies the condition of orbitally DS-convergence (or $X$ is orbitally DS-complete) iff: $\left\{T^{n} x\right\}_{n \in \mathbb{N} \cup\{0\}}$ for $x \in X$ is an arbitrary iteration sequence in $X$ and

$$
\sum_{n=0}^{\infty} \rho\left[T^{n} x, T^{n+1} x\right]=+\infty \quad(\text { for } x \in X)
$$

implies that $\left\{T^{n} x\right\}_{n \in \mathbb{N} \cup\{0\}}$ has a convergent subsequence in $X$.

We are now in a position to formulate our main statements (Geometric statements of fixed point) in the following forms.

Theorem 1. Let $T$ be a self-map on an orbitally DS-complete lower transversal space $(X, \rho)$. Suppose that there exists an upper semicontinuous function 
$G: X \rightarrow \mathbb{R}$ such that

$$
\rho(x, T(x)) \geq G(T x)-G(x) \geq 0
$$

for every $x \in X$. If $G\left(T^{n} a\right) \rightarrow+\infty$ as $n \rightarrow \infty$ for some $a \in X$, then $T$ has a fixed point in $X$.

Proof. Let $x \in X$ be an arbitrary point in $X$. We can show then that the sequence of iterates $\left\{T^{n} x\right\}_{n \in \mathbb{N} \cup\{0\}}$ in $X$ satisfies the condition of DS-convergence. In this sense, from (A) we have

$$
\sum_{i=0}^{n} \rho\left[T^{i} x, T^{i+1} x\right] \geq G\left(T^{n+1} x\right)-G(x),
$$

and thus, from the conditions for functional $G$, as $n \rightarrow \infty$, we obtain the following fact:

$$
\sum_{i=0}^{\infty} \rho\left[T^{i} a, T^{i+1} a\right] \geq \lim _{n \rightarrow \infty}\left(G\left(T^{n+1} a\right)-G(a)\right)=+\infty .
$$

Hence, for the subsequence of iterates $\left\{T^{n(k)} a\right\}_{k \in \mathbb{N} \cup\{0\}}$ in $X$, by orbitally DScompleteness, there is $\xi \in X$ such that $T^{n(k)} a \rightarrow \xi(k \rightarrow \infty)$. Since $\xi \in X$, from (A), we obtain the following inequality in the form

$$
\rho(\xi, T(\xi)) \geq G(T \xi)-G(\xi) \geq 0 ;
$$

i.e., since $G$ is an upper semicontinuous function, we have

$$
\rho(\xi, T(\xi)) \geq \limsup _{k \rightarrow \infty} G\left(T^{n(k)+1} a\right)-G(\xi)=+\infty ;
$$

which means, by the properties of a lower transversal space, $T \xi=\xi$. The proof is complete.

In connection with the preceding statement, we now show that the following facts hold.

Let $X$ be a topological space (or only a nonempty set), let $T: X \rightarrow X$ and let $A: X \times X \rightarrow \mathbb{R}_{+}^{0}$ be a given mapping. We shall introduce the concept of DA-variation in a space $X$, i.e., a topological space $X$ satisfies the condition of DA-variation iff: there exists a function $A: X \times X \rightarrow \mathbb{R}_{+}^{0}$ such that

$$
\sum_{n=0}^{\infty} A\left(T^{n} x, T^{n+1} x\right)=+\infty
$$

for arbitrary $x \in X$. In this case if (1) holds, we say and that $T: X \rightarrow X$ is unbounded variation or unbounded $A$-variation.

Lemma 1. Let $X$ be a nonempty set, $T: X \rightarrow X$, and let $A: X \times X \rightarrow \mathbb{R}_{+}^{0}$ be a given mapping. Then the following facts are mutually equivalent:

(a) $T$ is an unbounded variation mapping on a nonempty set $X$ in the sense of the mapping $A$. 
(b) There is an unbounded function $G: X \rightarrow \mathbb{R}_{+}^{0}$ such that holds the following inequality in the form

$$
A\left(T^{n} x, T^{n+1} x\right) \geq G(T x)-G(x) \geq 0
$$

for every $n \in \mathbb{N} \cup\{0\}$ and for every $x \in X$, where $G\left(T^{n} x\right) \rightarrow+\infty$ as $n \rightarrow \infty$.

(c) There is a nonnegative sequences of real functions in the form $x \mapsto C_{n}(x, T x)$ such that the following inequality holds

$$
A\left(T^{n} x, T^{n+1} x\right) \geq C_{n}(x, T x)
$$

for every $n \in \mathbb{N} \cup\{0\}$ and for every $x \in X$, where $\sum_{n=0}^{\infty} C_{n}(x, T x)=+\infty$ for arbitrary $x \in X$.

Proof. For the proof of this facts, first, suppose that holds (a), then we define the function $G: X \rightarrow \mathbb{R}_{+}^{0}$ by,

$$
G(x)=\sum_{i=0}^{n} A\left(T^{i} x, T^{i+1} x\right) \quad \text { for } \quad x \in X ;
$$

and thus we have $G(T x)-G(x) \leq A\left(T^{n} x, T^{n+1} x\right)$, i.e., the condition (b) holds. If (b) holds, we obtain that holds and the following inequality

$$
A\left(T^{n} x, T^{n+1} x\right) \geq G\left(T^{n+1} x\right)-G\left(T^{n} x\right)
$$

for every $x \in X$ and for every $n \in \mathbb{N} \cup\{0\}$. We set $C_{n}(x, T x)=G\left(T^{n+1} x\right)-G\left(T^{n} x\right)$ directly we obtain (c). Also, elementary, (a) is a consequence of (c). The proof is complete.

As a further application of the preceding ideas we have the following geometric statement.

Theorem 2. Let $T$ be a self-map on an orbitally DS-compete lower transversal space $(X, \rho)$. Suppose that there exists a lower semicontinuous function $G: X \rightarrow$ $\mathbb{R}_{+}^{0}$ such that

$$
\rho(x, T(x)) \geq \frac{1}{G(T x)}-\frac{1}{G(x)} \geq 0
$$

for every $x \in X$. If $G\left(T^{n} a\right) \rightarrow 0$ as $n \rightarrow \infty$ for some $a \in X$, then $T$ has a fixed point in $X$.

The proof of this statement is a totally analogous to the proof of Theorem 1 . Thus we the proof to leave out.

Lemma 2. Let $X$ be a nonempty set, $T: X \rightarrow X$, and let $A: X \times X \rightarrow \mathbb{R}_{+}^{0}$ be a given mapping. Then the following facts are mutually equivalent:

(a) $T$ is an unbounded variation mapping on a nonempty set $X$ in the sense of the mapping $A$.

(b') There is a function $G: X \rightarrow \mathbb{R}_{+}^{0}$ such that holds the following inequality in the form

$$
A\left(T^{n} x, T^{n+1} x\right) \geq \frac{1}{G(T x)}-\frac{1}{G(x)} \geq 0
$$

for every $n \in \mathbb{N} \cup\{0\}$ and for every $x \in X$, where $G\left(T^{n} x\right) \rightarrow 0$ as $n \rightarrow \infty$. 
Proof. For the proof of this statement suppose that holds (a). We define the function $G: X \rightarrow \mathbb{R}_{+}^{0}$ by

$$
G(x)=\frac{1}{\sum_{i=0}^{n} A\left(T^{i} x, T^{i+1} x\right)} \quad \text { for } \quad x \in X ;
$$

and thus we have $1 / G(T x)-1 / G(x) \leq A\left(T^{n} x, T^{n+1} x\right)$, i.e., the condition (b') holds. If (b') holds, then holds (c) in Lemma 1, and thus, from Lemma 1 we obtain (a). The proof is complete.

Annotation 1. Let $X$ be a topological space let $T: X \rightarrow X$, and let $A: X \times X \rightarrow \mathbb{R}_{+}^{0}$ be a given mapping. We shall introduce the concept of DS-convergence in a space $X$, i.e., a topological space $X$ satisfies the condition of DS-convergence iff: $\left\{x_{n}\right\}_{n \in \mathbb{N}}$ is an arbitrary sequence in $X$ and $\sum_{i=1}^{\infty} A\left(x_{i}, x_{i+1}\right)=+\infty$ implies that $\left\{x_{n}\right\}_{n \in \mathbb{N}}$ has a convergent subsequence in $X$.

On the other hand, a topological space $X$ satisfies the following condition of orbitally DS-convergence iff: $\left\{T^{n} x\right\}_{n \in \mathbb{N} \cup\{0\}}$ for $x \in X$ is an arbitrary iteration sequence in $X$ and $\sum_{n=0}^{\infty} A\left(T^{n} x, T^{n+1} x\right)=+\infty$ (for $\left.x \in X\right)$ implies that $\left\{T^{n} x\right\}_{n \in \mathbb{N} \cup\{0\}}$ has a convergent subsequence in $X$.

We are now in a position to formulate our main geometric statements of fixed point on arbitrary topological spaces.

Theorem 1a. Let $T$ be a self-map on a topological space $X$ which is with the property of orbitally DS-convergence. Suppose that there exists an upper semicontinuous function $G: X \rightarrow \mathbb{R}$ such that

$$
A(x, T(x)) \geq G(T x)-G(x) \geq 0
$$

for every $x \in X$. If $A(a, b)=+\infty$ iff $a=b$ and if $G\left(T^{n} z\right) \rightarrow+\infty(n \rightarrow \infty)$ for some $z \in X$, then $T$ has a fixed point in $X$.

The proof of this statement is a totally analogous with the proof of Theorem 1.

Theorem 2a. Let $T$ be a self-map on a topological space $X$ which is with the property of orbitally DS-convergence. Suppose thath there exists a lower semicontinuous function $G: X \rightarrow$ $\mathbb{R}_{+}^{0}$ such that

$$
A(x, T(x)) \geq \frac{1}{G(T x)}-\frac{1}{G(x)} \geq 0
$$

for every $x \in X$. If $A(a, b)=+\infty$ iff $a=b$ and if $G\left(T^{n} z\right) \rightarrow+\infty(n \rightarrow \infty)$ for some $z \in X$, then $T$ has a fixed point in $X$.

The proof of this statement is a totally analogous with the proof of Theorem 2 .

In connection with the preceding, we shall introduce the concept of lower topological space. In this sense, the function $A: X \times X \rightarrow[0,+\infty]$ is called a lower transverse on a nonempty set $X$ (or lower transversal) iff: $A(x, y)=+\infty$ if and only if $x=y$ for all $x, y \in X$.

A lower topological space $(X, A)$ is a topological space $X$ together with a given lower transverse $A$ on $X$.

Otherwise, the function $A$ is called a semilower transverse on a nonempty set $X$ iff: $A(x, y)=+\infty$ implies $x=y$ for all $x, y \in X$. A semilower topological space $(X, A)$ is a topological sapce $X$ together with a given semilower transverse $A$ on $X$.

We notice, in connection with the preceding, that Theorems 1a and 2 a de facto on lower topological spaces hold.

Theorem 1b. Let $T$ be a self-map on a semilower topological space $(X, A)$ which is with the property of DS-convergence. Suppose that there exists an unbounded upper semicontinuous function $G: X \rightarrow \mathbb{R}_{+}^{0}$ such that

$$
A(x, y) \geq G(y)-G(x)
$$

for all $x, y \in X$. Then $T$ has a fixed point in $X$. 
Proof. From the facts of this statement there exists a sequence $\left\{x_{n}\right\}_{n \in \mathbb{N}}$ in $X$ such that $G\left(x_{n}\right) \rightarrow+\infty$ as $n \rightarrow \infty$. On the other hand, from (N), we have

$$
\sum_{n=1}^{\infty} A\left(x_{n}, x_{n+1}\right) \geq \sum_{n=1}^{\infty}\left(G\left(x_{n+1}\right)-G\left(x_{n}\right)\right)=+\infty,
$$

and thus, by DS-completeness, there is $\zeta \in X$ such that $x_{n(k)} \rightarrow \zeta(k \rightarrow \infty)$. Since $\zeta \in X$, from $(\mathrm{N})$, we obtain the following inequality in the form

$$
A(\zeta, T(\zeta)) \geq G(\zeta)-G(T \zeta)
$$

i.e., since $G$ is an upper semicontinuous function, we have the following inequality and equality of the form

$$
A(\zeta, T(\zeta)) \geq \limsup _{k \rightarrow \infty} G\left(x_{n(k)}\right)-G(T \zeta)=+\infty
$$

which means, by properties of a semilower topological space, $T(\zeta)=\zeta$. The proof is complete.

We notice that parallely with the preceding statements we have and the following results on the lower transversal spaces. This results are not dually with the preceding results!

Theorem 3. Let $T$ be a self-map on an orbitally DS-complete lower transversal space $(X, \rho)$. Suppose that there exists a lower semicontinuous function $G: X \rightarrow \mathbb{R}$ such that

$$
\rho(x, T(x)) \geq G(x)-G(T x) \geq 0
$$

for every $x \in X$. If $G\left(T^{n} a\right) \rightarrow-\infty$ as $n \rightarrow \infty$ for some $a \in X$ or if $G$ is unbounded, then $T$ has a fixed point in $X$.

The proof of this statement is a totally analogous with the proof of the former Theorem 1.

We notice that inequality (A) is not dually, in comparable, with inequality (B). This implies that results of Theorems 1 and 3 are independents.

Lemma 3. Let $X$ be a nonempty set, $T: X \rightarrow X$, and let $A: X \times X \rightarrow \mathbb{R}_{+}^{0}$ be a given mapping. Then the following facts are mutually equivalent:

(a) $T$ is an unbounded variation mapping on a nonempty set $X$ in the sense of the mapping $A$.

(b) There is an unbounded function $G: X \rightarrow \mathbb{R}$ such that holds the following inequality in the form

$$
A(x, T x) \geq G(x)-G(T x) \geq 0
$$

for every $x \in X$, where $G\left(T^{n} x\right) \rightarrow-\infty$ as $n \rightarrow \infty$.

The proof of this statement is an analogous with the proofs of the former Lemmas 1 and 2 .

Theorem 4. Let $T$ be a self-map on an orbitally DS-complete lower transversal space $(X, \rho)$. Suppose that there exists an upper semicontinuous function $G: X \rightarrow$ $\mathbb{R}_{-}:=(-\infty, 0)$ such that

$$
\rho(x, T(x)) \geq \frac{1}{G(x)}-\frac{1}{G(T x)} \geq 0
$$


for every $x \in X$. If $G\left(T^{n} a\right) \rightarrow 0$ as $n \rightarrow \infty$ for some $a \in X$, then $T$ has a fixed point in $X$.

The proof of this statement we to leave out. Because the proof a totally analogous with the former statements of Theorems 1,2 and 3 .

Lemma 4. Let $X$ be a nonempty set, $T: X \rightarrow X$, and let $A: X \times X \rightarrow \mathbb{R}_{+}^{0}$ be a given mapping. Then the following facts are mutually equivalent:

(a) $T$ is an unbounded variation mapping on a nonempty set $X$ in the sense of the mapping $A$.

(b) There is a function $G: X \rightarrow \mathbb{R}_{-}^{0}$ such that holds the following inequality in the form

$$
A\left(T^{n} x, T^{n+1} x\right) \geq \frac{1}{G(x)}-\frac{1}{G(T x)} \geq 0
$$

for every $n \in \mathbb{N} \cup\{0\}$ and for every $x \in X$, where $G\left(T^{n} x\right) \rightarrow 0$ as $n \rightarrow \infty$.

The proof of this statement is a totally analogous with the proof of Lemma 2 . Thus the proof we to leave out.

Annotation 2. In this part of paper we are now in a position to formulate two geometric statements of fixed point on arbitrary topological spaces.

Theorem 3a. Let $T$ be a self-map on a topological space $X$ which is with the property of orbitally DS-convergence. Suppose that there exists a lower semicontinuous function $G: X \rightarrow \mathbb{R}$ such that

$$
A(x, T(x)) \geq G(x)-G(T x) \geq 0
$$

for every $x \in X$. If $A(a, b)=+\infty$ iff $a=b$ and if $G\left(T^{n} z\right) \rightarrow-\infty(n \rightarrow \infty)$ for some $z \in X$, then $T$ has a fixed point in $X$.

The proof of this statement is a totally analogous with the proof of Theorem 3 .

Theorem 4a. Let $T$ be a self-map on a topological space $X$ which is with the property of orbitally DS-convergence. Suppose that there exists an upper semicontinuous function $G: X \rightarrow$ $\mathbb{R}_{-}$such that

$$
A(x, T(x)) \geq \frac{1}{G(x)}-\frac{1}{G(T x)} \geq 0
$$

for every $x \in X$. If $A(a, b)=+\infty$ iff $a=b$ and if $G\left(T^{n} z\right) \rightarrow 0(n \rightarrow \infty)$ for some $z \in X$, then $T$ has a fixed point in $X$.

The proof of this statement is a totally analogous with the proof of Theorem 4.

Annotation 3. We notice that in [17] Tasković proved the following statement for a class of expansion mappings. Namely, if $(X, \rho)$ is an orbitally DS-complete lower transversal space, if $T: X \rightarrow X$, and if there exists a number $q>1$ such that

$$
\rho(T(x), T(y)) \geq q \rho(x, y)
$$

for each $x, y \in X$, then $T$ has a unique fixed point in the lower transversal space $X$.

Annotation 4. Let $\left(X, \rho_{X}\right)$ and $\left(Y, \rho_{Y}\right)$ be two lower transversal spaces and let $T: X \rightarrow Y$.

We notice, from: Tasković [17], that $T$ be lower continuous at $x_{0} \in X$ iff for every $\varepsilon>0$ there exists a $\delta>0$ such that the relation

$$
\rho_{X}\left[x, x_{0}\right]>\delta \quad \text { implies } \rho_{Y}\left[T(x), T\left(x_{0}\right)\right]>\varepsilon .
$$

A typical first example of a lower continuous mapping is the mapping $T: X \rightarrow X$ with property (2). For further facts on the lower continuous mappings see: Task ović [17]. 
Annotation 5. For any nonempty set $S$ in the lower transversal space $X$ the diameter of $S$ is defined as

$$
\operatorname{diam}(S):=\inf \{\rho[x, y]: x, y \in S\}
$$

where $A \subset B$ implies $\operatorname{diam}(B) \leq \operatorname{diam}(A)$. The relation $\operatorname{diam}(S)=+\infty$ holds if and only if $S$ is a one point set.

Elements of a lower transversal space will usually be called points. Given a lower transversal space $(X, \rho)$, with the bisection function $d:[0,+\infty]^{2} \rightarrow[0,+\infty]$ and a point $z \in X$, the open ball of center $z$ and radius $r>0$ is the set

$$
d(B(z, r))=\{x \in X: \rho[z, x]>r\} .
$$

On the other hand, from Tasković [17], the convergence $x_{n} \rightarrow x$ as $n \rightarrow \infty$ in the lower transversal space $(X, \rho)$ means that

$$
\rho\left[x_{n}, x\right] \rightarrow+\infty \quad \text { as } n \rightarrow \infty,
$$

or equivalently, for every $\varepsilon>0$ there exist an integer $n_{0}$ such that the relation $n \geq n_{0}$ implies $\rho\left[x_{n}, x\right]>\varepsilon$.

The sequence $\left\{x_{n}\right\}_{n \in \mathbb{N}}$ in the lower transversal space $(X, \rho)$ is called transversal sequence (or lower Cauchy sequence) iff for every $\varepsilon>0$ there is an $n_{0}=n_{0}(\varepsilon)$ such that

$$
\rho\left[x_{n}, x_{m}\right]>\varepsilon \text { for all } n, m \geq n_{0} .
$$

Let $(X, \rho)$ be a lower transversal space and $T: X \rightarrow X$. We notice, from Tasković [17], that a sequence of iterates $\left\{T^{n}(x)\right\}_{n \in \mathbb{N}}$ in $X$ is said to be transversal sequence if and only if

$$
\lim _{n \rightarrow \infty}\left(\operatorname{diam}\left\{T^{k}(x): k \geq n\right\}\right)=+\infty \text {. }
$$

In this sense, a lower transversal space is called lower complete iff every transversal sequence converges.

Also, a space $(X, \rho)$ is said to be lower orbitally complete (or lower T-orbitally complete) iff every transversal sequence which in contained in $\mathcal{O}(x):=\left\{x, T x, T^{2}(x), \ldots\right\}$ for some $x \in X$ converges in $X$.

Theorem 5. (Tasković [17]). Let $T$ be mapping of a lower transversal space $(X, \rho)$ into itself and let $X$ be lower $T$-orbitally complete. Suppose that there exists a function $\varphi:[0,+\infty] \rightarrow[0,+\infty]$ satisfying

$$
\left.\left(\forall t \in \mathbb{R}_{+}^{0}\right)\left(\varphi(t)>t \quad \text { and } \quad \liminf _{z \rightarrow t-0} \varphi(z)>t\right)\right)
$$

such that

$$
\rho[T x, T y] \geq \varphi\left(\operatorname{diam}\left\{x, y, T x, T y, T^{2} x, T^{2} y, \ldots\right\}\right)
$$

for all $x, y \in X$. If $x \mapsto \operatorname{diam} \mathcal{O}(x)$ or $x \mapsto \rho[x, T x]$ is T-orbitally upper semicontinuous, then $T$ has a unique fixed point $\zeta \in X$, and $\left\{T^{n}(x)\right\}_{n \in \mathbb{N}}$ converges to $\zeta$ for every $x \in X$.

A variant brief proof of this statement may be found in: Tasković [17] with application of the following context.

Lemma 5. (Tasković $[17])$. Let the mapping $\varphi:[0,+\infty] \rightarrow[0,+\infty]$ have the property (Id). If the sequence $\left(x_{n}\right)$ of nonnegative real numbers satisfies the condition

$$
x_{n+1} \geq \varphi\left(x_{n}\right), \quad n \in \mathbb{N},
$$

then the sequence $\left(x_{n}\right)$ tends to $+\infty$. The velocity of this convergence is not necessarily geometrical. 
A brief first proof of this fact may be found in: Tasković [14]. A brief second proof of this statement may be found in Tasković [6].

As immediate consequences of the preceding Theorem 5 we obtain directly the following interesting cases of $(\mathrm{D})$ :

(a) There exists a nondecreasing function $\varphi:[0,+\infty] \rightarrow[0,+\infty]$ satisfying $\liminf _{z \rightarrow t-0} \varphi(z)>t$ for every $t \in \mathbb{R}_{+}^{0}$ such that

$$
\rho[T x, T y] \geq \varphi(\operatorname{diam}\{x, y, T x, T y\})
$$

for all $x, y \in X$.

(b) (Special linear case of (D) for $\varphi(t)=\alpha t$ ). There exists a constant $\alpha>1$ such that for all $x, y \in X$ the following inequality holds

$$
\rho[T x, T y] \geq \alpha \operatorname{diam}\{x, y, T x, T y\},
$$

i.e., equivalently to

$$
\rho[T x, T y] \geq \alpha \min \{\rho[x, y], \rho[x, T x], \rho[y, T y], \rho[x, T y], \rho[y, T x]\} .
$$

(c) There exists a nondecreasing function $\varphi:[0,+\infty] \rightarrow[0,+\infty]$ satisfying the following fact that $\liminf \operatorname{int}_{z \rightarrow 0} \varphi(z)>t$ for every $t \in \mathbb{R}_{+}^{0}$ such that

$$
\rho[T x, T y] \geq \varphi\left(\operatorname{diam}\left\{x, y, T x, T y, \ldots, T^{k} x, T^{k} y\right\}\right)
$$

for an arbitrary fixed integer $k \geq 0$ and for all $x, y \in X$.

(d) There exists an increasing mapping $f:[0,+\infty]^{5} \rightarrow[0,+\infty]$ (i.e., $x_{i} \leq y_{i}(i=1, \ldots, 5)$ implies that we have $\left.f\left(x_{1}, \ldots, x_{5}\right) \leq f\left(y_{1}, \ldots, y_{5}\right)\right)$ satisfying the following inequality in the form $\liminf _{z \rightarrow t-0} f(z, z, z, z, z)>t$ for every $t \in \mathbb{R}_{+}^{0}$ such that

$$
\rho[T x, T y] \geq f(\rho[x, y], \rho[x, T x], \rho[y, T y], \rho[x, T y], \rho[y, T x])
$$

for all $x, y \in X$.

Convexity on lower transversal spaces. The set $C$ in linear space is lower convex if for $x, y \in C$ and $\lambda \in[1,2]$ implies that $\lambda x+(1-\lambda) y \in C$. The lower transversal space $(X, \rho)$ is called lower convex (or transversal lower convex) if for any two different points $x, y \in X$ there is a point $z \in X(z \neq x, y)$ such that

$$
\rho[x, y]+\rho[y, z]=\frac{9}{2} \rho[x, z] .
$$

In connection with this, if $C \subset X$ is a lower convex set of a transversal lower normed space $X$, then $C$ also and transversal lower convex space with $\rho[x, y]=$ $1 /\|x-y\|$ for the classical norm $\|$.$\| , because for any two different points x, y \in C$ there is a point $z:=(3 y-x) / 2 \in C(z \neq x, y)$ such that $(\mathrm{Cd})$ holds. For further facts on lower transversal normed spaces see: Tasković [17].

We are now in a position to formulate the following general statements which are a based for geometry (lower convexity) of lower transversal spaces. The following statements are very connection with the famous Schauder's problem (Scottish book, problem 54), from: Tasković [12].

Proposition 1. Let $C$ be a nonempty lower convex compact subset of a linear topological space $X$ and suppose $T: C \rightarrow C$ is a lower continuous mapping. Then $T$ has a fixed point in $C$. 
A suitable brief proof of this statement may be found in Tasković [17]. Also, a proof of this statement we can give and from the preceding facts of this paper.

Proposition 2. Suppose that $C$ is a nonempty lower convex lower compact subset of $\mathbb{R}^{n}$, and that $T: C \rightarrow C$ is a lower continuous mapping. Then $T$ has a fixed point in $C$.

We can now formulate Proposition 2 in a manner valid for all transversal lower normed linear spaces.

Proposition 3. Let $C$ be a nonempty, lower compact, lower convex subset of a transversal lower normed space $X$, and suppose $T: C \rightarrow C$ is a lower continuous operator. Then $T$ has a fixed point in $C$.

We notice that Propositions 2 and 3 are directly consequences of Proposition 1. A brief suitable proof of Proposition 3 may be found in Tasković [17].

On the other hand, in connection with the preceding facts, the set $C$ in linear space is convex if for $x, y \in C$ and $\lambda \in[0,1]$ implies $\lambda x+(1-\lambda) y \in C$.

The lower transversal space $(X, \rho)$ is called $\mathbf{D}$-convex (or transversal $D$-convex) if for two different points $x, y \in X$ there is a point $z \in X(z \neq x, y)$ such that

$$
\rho[x, y]+\rho[y, z]=\rho[x, z] .
$$

In connection with this, if $C \subset X$ is a convex set of a transversal lower normed space $X$, then $C$ also and transversal D-convex space with $\rho[x, y]=1 /\|x-y\|$ for the classical norm $\|\cdot\|$, because for any two different points $x, y \in C$ there is a point

$$
z:=\frac{3-\sqrt{5}}{2} y+\frac{\sqrt{5}-1}{2} x \in C \quad(z \neq x, y)
$$

such that (Dc) holds. For further facts of this see: Taskovic [17].

Proposition 4. Let $C$ be a nonempty convex compact subset of a linear topological space $X$ and suppose $T: C \rightarrow C$ is a lower continuous mapping. Then $T$ has a fixed point in $C$.

A proof of this statement we can give from the preceding facts of this paper. The proof of this statement is similary with the proof of the former Proposition 1.

Proposition 5. Suppose that $C$ is a nonempty $D$-convex lower compact subset of $\mathbb{R}^{n}$, and that $T: C \rightarrow C$ is a lower continuous mapping. Then $T$ has a fixed point in $C$.

From Proposition 4 we can now formulate Proposition 5 in a manner valid for all transversal lower normed linear spaces.

Proposition 6. Let $C$ be a nonempty, lower compact, D-convex subset of a transversal lower normed space $X$, and suppose $T: C \rightarrow C$ is a lower continuous operator. Then $T$ has a fixed point in $C$. 
We notice that Propositions 5 and 6 are directly consequences of Proposition 4. A brief suitable proof of Proposition 6 may be found in Tasković [17].

Open problem 1. Does every lower continuous mapping of compact set $C \subset$ $X$ into itself in linear topological space $X$ has a fixed point in $C$, where $C$ with the property that: $\lambda x+(1-\lambda) y \in C$ for all $x, y \in C$ and $\lambda \in[n, n+1]$ for an arbitrary fixed number $n \in \mathbb{N}$ !?

We notice that the cases $n=0$ and $n=1$, of this problem, are solve via Propositions 1 and 4 of this paper.

A characterization of the class of lower contraction mappings. In this part of this paper we introduce the concept of lower transversal contraction mapping $T$ of a transversal lower space $X$, i.e., of a mapping $T: X \rightarrow X$ such that for all $x, y \in X$ there exists a sequence of nonnegative real functions $\mathcal{D}_{n, r}(x, y)$ with $\mathcal{D}_{n, r}(x, y) \rightarrow+\infty(r \geq n \rightarrow \infty)$ and positive integer $m(x, y)$ such that

$$
\rho\left[T^{n} x, T^{r} y\right] \geq \mathcal{D}_{n, r}(x, y) \text { for all } r>n \geq m(x, y) .
$$

Also, in this section we introduced the concept of lower $\sigma$-contraction $T$ of a lower transversal space $X$ into itself, i.e., of a mapping $T: X \rightarrow X$ such that for all $x, y \in X$ there exist numbers $C_{n}(x, y)>0$ and $K(x, y)>0$ such that

$$
\rho\left[T^{n} x, T^{n} y\right] \geq C_{n}(x, y) K(x, y), \quad \text { for } n \in \mathbb{N},
$$

where $\inf \left\{C_{n}(x, y), C_{n+1}(x, y), \ldots\right\} \rightarrow+\infty$ as $n \rightarrow \infty$ for all $x, y \in X$.

A mapping $T$ of a lower transversal space $X$ into itself is said to be lower orbitally continuous if $T$ for every $x \in X$ a lower continuous mapping via orbits, i.e., if $T\left(T^{n(k)} x\right) \rightarrow T \zeta$ whenever $T^{n(k)} x \rightarrow \zeta \in X$ for every $x \in X$ and $k \in \mathbb{N}$.

We will in further denote by $\mathcal{F}([0,+\infty])$ the set of all lower bisection functions $d:[0,+\infty]^{2} \rightarrow[0,+\infty]$ which are increasing satisfying $d(t, t) \geq t$ for every $t \in[0,+\infty]$.

Proposition 7. Necessary and sufficient conditions that a mapping $T$ of a lower transversal space $(X, \rho)$ into itself with a bisection function $d \in \mathcal{F}([0,+\infty])$ and with the lower continuity of $\rho$ has the following properties: (a) $T$ has a unique fixed point $\zeta \in X$, (b) $x_{n}=T^{n}(x) \rightarrow \zeta(n \rightarrow \infty)$ for arbitrary $x \in X$, and

$$
\rho\left[T^{n} x, \zeta\right] \geq \mathcal{D}_{n}(x, T x), \quad \text { for } \quad n \geq m(x),
$$

where $x \mapsto \mathcal{D}_{n}(x, T x)$ are real nonnegative functions with $\mathcal{D}_{n}(x, T x) \rightarrow+\infty(n \rightarrow$ $\infty)$ are the following ones: (e) $X$ is lower $T$-orbitally complete, ( $f$ ) $T$ is lower orbitally continuous, and $(g) T$ is lower transversal contraction mapping where

$$
\mathcal{D}_{n, r}(x, y)=\min \left\{\mathcal{D}_{n}(x, T x), \mathcal{D}_{r}(y, T y)\right\}
$$

The proof of this statement is very similar with the proof suitable for the metric spaces. For this see: Tasković [5] and [17]. 
Proposition 8. Let $(X, \rho)$ be a lower transversal space with the lower continuity of $\rho$ and with a bisection function $d \in \mathcal{F}([0,+\infty])$ and $T$ a map of $X$ into itself. The conditions (a), (b) of proposition 7, and for each $x \in X$

$$
\rho\left[T^{n} x, \zeta\right] \geq K(x, T x) \inf \left\{C_{n}(x, T x), C_{n+1}(x, T x), \ldots\right\}, \quad \text { for } n \in \mathbb{N},
$$

are necessary for the conditions (f), (e) of proposition 7 , and lower $\sigma$-contraction.

On the other hand, the conditions (a), (b) of proposition 7, and ( $\left.d_{1}\right)$ with $\inf \left\{C_{n}(x, T x), C_{n+1}(x, T x), \ldots\right\} \rightarrow+\infty$ as $n \rightarrow \infty$ for every $x \in X$ are sufficient for $(f)$, (e) of proposition $\%$, and lower $\sigma$-contraction.

Now we shall show how Proposition 8 can be applied for investigation of solvability of an integral equation of the form

$$
u(x)=v(x)+\lambda \int_{\alpha}^{x} K(x, t, u(t)) d t, \quad \text { for } \quad \alpha \leq x \leq \beta,
$$

by successive approximation, where $\lambda \in \mathbb{R}$ is an arbitrary parameter, $v(x)$ is a given lower continuous function in $[\alpha, \beta]$ and $K(m, s, x)$ is continuous or lower continuous for $m, s \in[\alpha, \beta]$. The $L C[\alpha, \beta]$ denote the set of all lower continuous functions on $[\alpha, \beta]$.

Proposition 9. Let $K:[\alpha, \beta] \times[\alpha, \beta] \times \mathbb{R} \rightarrow \mathbb{R}$ be continuous or lower continuous and let satisfy the following Lipschitz condition of the form

$$
|K(m, s, x)-K(m, s, y)| \leq L|x-y|, \quad L=\text { const. }
$$

for all $(m, s) \in[\alpha, \beta] \times[\alpha, \beta]$ and $x, y \in \mathbb{R}$. Then for any $v \in L C[\alpha, \beta]$ the equation (IE) has a unique solution $u \in L C[\alpha, \beta]$. Moreover, defining a sequence of functions $\left\{u_{n}\right\}_{n \in \mathbb{N}}$ inductively by choosing any $u_{0} \in L C[\alpha, \beta]$ and setting

$$
u_{n+1}(x)=v(x)+\lambda \int_{\alpha}^{x} K\left(x, t, u_{n}(t)\right) d t,
$$

the sequence $\left\{u_{n}\right\}_{n \in \mathbb{N}}$ lower converges (lower uniformly) on $[\alpha, \beta]$ to the unique solution $u$ of the equation (IE).

\section{FiXed POINTS ON UPPER TRANSVERSAL SPACES}

Let $(X, \rho)$ be an upper transversal space and $T: X \rightarrow X$. We shall introduce the concept of CS-convergence in a space $X$; i.e., an upper transversal space $X$ satisfies the condition of CS-convergence (or $X$ is CS-complete) iff: $\left\{x_{n}\right\}_{n \in \mathbb{N}}$ is an arbitrary sequence in $X$ and $\sum_{i=1}^{\infty} \rho\left[x_{i}, x_{i+1}\right]<+\infty$ implies that $\left\{x_{n}\right\}_{n \in \mathbb{N}}$ has a convergent subsequence in $X$.

Also, an upper transversal space $X$ satisfies the condition of orbitally CSconvergence (or $X$ is orbitally CS-complete) iff: $\left\{T^{n} x\right\}_{n \in \mathbb{N} \cup\{0\}}$ for $x \in X$ is an arbitrary iteration sequence in $X$ and $\sum_{n=0}^{\infty} \rho\left[T^{n} x, T^{n+1} x\right]<+\infty$ (for $x \in X$ ) implies that $\left\{T^{n} x\right\}_{n \in \mathbb{N} \cup\{0\}}$ has a convergent subsequence in $X$.

We are now in a position to formulate our main statements (Geometric statements of fixed point) on the upper transversal spaces. 
This part of paper continues the study of the preceding results based on a new geometry on upper transversal spaces.

Theorem 6. Let $T$ be a self-map on an orbitally CS-complete upper transversal space $(X, \rho)$. Suppose that there exists a bounded above function $G: X \rightarrow \mathbb{R}$ such that

$$
\rho[x, T x] \leq G(T x)-G(x)
$$

for every $x \in X$. If $x \mapsto \rho[x, T x]$ is a lower semicontinuous function, then $T$ has a fixed point in $X$.

Proof. Let $x$ be an arbitrary point in $X$. We can show then that the sequence of iterates $\left\{T^{n} x\right\}_{n \in \mathbb{N} \cup\{0\}}$ in $X$ satisfies the condition of CS-convergence. In this sense, from (E) we have

$$
\sum_{i=0}^{n} \rho\left[T^{i} x, T^{i+1} x\right] \leq G\left(T^{n+1} x\right)-G(x),
$$

and thus, since $G$ is a bounded functional, we obtain, as $n \rightarrow \infty$ the following fact in the form

$$
\sum_{i=0}^{\infty} \rho\left[T^{i} x, T^{i+1} x\right]<+\infty \quad \text { for } \quad x \in X
$$

Hence, for the subsequence of iterates $\left\{T^{n(k)} x\right\}_{k \in \mathbb{N} \cup\{0\}}$ in $X$, by orbitally CScompleteness, there is $\zeta \in X$ such that $T^{n(k)} x \rightarrow \zeta(k \rightarrow \infty)$. Since $x \mapsto \rho[x, T x]$ is a lower semicontinuous function at $\zeta$,

$$
\rho[\zeta, T \zeta] \leq \liminf _{k \rightarrow \infty} \rho\left[T^{n(k)} x, T^{n(k)+1} x\right]=0 .
$$

Thus $T \zeta=\zeta$, and we have shown that for each $x \in X$ the subsequence $\left\{T^{n(k)} x\right\}_{k \in \mathbb{N} \cup\{0\}}$ converges to a fixed point $\zeta$ of $T$. This completes the proof.

Annotation 6. We remark that the existence of fixed point for a contractive map $T$ in a CScomplete upper transversal space $(X, \rho)$ is a consequence of Theorem 6 ; for if $\rho[T x, T y] \leq \alpha \rho[x, y]$ with $0 \leq \alpha<1$, we have $\rho\left[T x, T^{2} x\right] \leq \alpha \rho[x, T x]$, therefore

$$
\rho[x, T x]-\alpha \rho[x, T x] \leq \rho[x, T x]-\rho\left[T x, T^{2} x\right]
$$

so, with the function $G(x):=(\alpha-1)^{-1} \rho[x, T x]$, the conditions of Theorem 6 are satisfied.

As an immediate application of the preceding statement, as a directly consequence, we obtain the following statement.

Theorem 7. Let $T$ be a self-map on an orbitally CS-complete upper transversal space $(X, \rho)$. Suppose that there exist a bounded above function $G: X \rightarrow \mathbb{R}$ such that

$$
\rho[x, T x] \leq \sum_{i=0}^{\infty}\left(G\left(T^{2 i+1} x\right)-G\left(T^{2 i} x\right)\right)
$$

and $G\left(T^{2 i} x\right) \leq G\left(T^{2 i+1} x\right)$ for $i \in \mathbb{N} \cup\{0\}$ and for every $x \in X$. If $x \mapsto \rho[x, T x]$ is a lower semicontinuous function, then $T$ has a fixed point in $X$. 
The proof of this statement is an analogous with the preceding proof of Theorem 6.

We notice that the proof of Theorem 6 is given in the form without Axiom of Choice. But, the following variant of Theorem 6 we give via Zorn's lemma in the following form.

Theorem 8. Let $T$ be a self-map on an orbitally CS-complete upper transversal space $(X, \rho)$. Suppose that there exists an upper semicontinuous bounded above function $G: X \rightarrow \mathbb{R}$ such that

$$
\rho[x, T x] \leq G(T x)-G(x)
$$

for every $x \in X$. Then $T$ has a fixed point in $X$.

A part of the proof for this statement is analogous to the proof of Theorem 6. A brief variant proof of this statement based on the preceding facts and $D$-Ordering Principle (dually form) may be found in: Tasković [6].

Proof of Theorem 8. (Application of Zorn's lemma). Define a relation $\preccurlyeq_{G, \rho}$ on $X$ by the following condition:

$$
a \preccurlyeq G, \rho \quad \text { if and only if } \rho[a, b] \leq G(b)-G(a) .
$$

It is to verify that $\preccurlyeq G, \rho$ is a partial ordering (asymmetric and transitive relation) in $X$. The space $X$ together with this partial ordering is denoted by $X_{G, \rho}$.

Fix $t \in X$ and use Zorn's lemma to obtain a maximal (relative to set inclusion) chain $M$ of $X_{G, \rho}$ containing $t$. Let $M:=\left\{x_{\alpha}\right\}_{\alpha \in I}$ and $x_{\alpha} \preccurlyeq G, \rho x_{\beta}$ if and only if $\alpha \leq \beta(\alpha, \beta \in I)$, where $I$ is totally ordered.

Now $\left\{G\left(x_{\alpha}\right)\right\}_{\alpha \in I}$ is an increasing net bounded above in $\mathbb{R}$, so there exists $r \in \mathbb{R}$ such that $G\left(x_{\alpha}\right) \rightarrow r$ as $\alpha \uparrow \infty$. Thus, as in the proof of Theorem 6 , we obtain that $\left\{x_{\alpha}\right\}_{\alpha \in I}$ is a transversal net in $X$.

By orbitally CS-completeness there is $x \in X$ such that $x_{\alpha} \rightarrow x$ as $\alpha \uparrow \infty$. Since $G$ is upper semicontinuous we obtain that is $\lim \cdot \sup G\left(x_{\alpha}\right) \leq G(x)$. Also, for $\alpha \leq \beta$,

$$
\rho\left[x_{\alpha}, x_{\beta}\right] \leq G\left(x_{\alpha}\right)-G\left(x_{\beta}\right),
$$

and letting $\beta \uparrow \infty, \rho\left[x_{\alpha}, x\right] \leq G(x)-G\left(x_{\alpha}\right)$ yielding $x_{\alpha} \preccurlyeq G, \rho$ for $\alpha \in I$. Since $M$ is a maximal chain, we have $x \in M$. On the other hand, also, $(\mathrm{H})$ holds so it follows that

$$
x_{\alpha} \preccurlyeq G, \rho=\preccurlyeq_{G, \rho} T x \quad \text { for } \quad \alpha \in I,
$$

and, by maximality, $T x \in M$. Therefore $T x \preccurlyeq G, \rho x$ and it follows that $T x=x$. The proof is complete.

Open problem 2. We notice that the preceding proof of Theorem 8 is given via Zorn's lemma. Does a new proof of Theorem 8 can be given elementary without Axiom of Choice?!

Some remarks. We notice that the preceding statements we can modify in the following sense. Naimely, the next statement follows from Theorem 6 as follows. 
Theorem 6a. Let $T$ be a self-map on an orbitally CS-complete upper transversal space $(X, \rho)$. Suppose that there exists a bounded above function $G: X \rightarrow \mathbb{R}$ such that for any $x \in X$, with $x \neq T x$, there exists $y \in X \backslash\{x\}$ with property

$$
\rho[x, y] \leq G(y)-G(x) .
$$
$X$.

If $x \mapsto \rho[x, T x]$ is a lower semicontinuous function, then $T$ has a fixed point in

On the other hand, as an immediately consequence of Theorem 8, we obtain the following fact as follows.

Theorem 8a. Let $T$ be a self-map on an orbitally CS-complete upper transversal space $(X, \rho)$. Suppose that there exists an upper semicontinuous bounded above function $G: X \rightarrow \mathbb{R}$ such that for any $x \in X$, with $x \neq T x$, there exists $y \in X \backslash\{x\}$ with property (K). Then $T$ has a fixed point in $X$.

A brief suitable proof of this statement based on Zorn's lemma may be found in Tasković [9].

We are now in a position to formulate an extension of Theorem 8 in the sense of a general condition which is a widening inequality (E).

Theorem 9. Let $T$ be a self-map on an orbitally CS-complete upper transversal space $(X, \rho)$ Suppose that there exists an upper semicontinuous bounded above function $G: X \rightarrow \mathbb{R}$ such that

$$
\rho[x, T x] \leq \sum_{i=0}^{\infty}\left(G\left(T^{2 i+1} x\right)-G\left(T^{2 i} x\right)\right)
$$

and $G\left(T^{2 i} x\right) \leq G\left(T^{2 i+1} x\right)$ for $i \in \mathbb{N} \cup\{0\}$ and for every $x \in X$. Then $T$ has a fixed point in $X$.

A brief suitable proof of this statement based on Zorn's lemma may be found in Tasković [13].

As an immediate consequence of the preceding result we obtain the following statement of fixed point.

Corollary 1. Let $T$ be a self-map on an orbitally CS-complete upper transversal space $(X, \rho)$. Suppose that there exist an upper semicontinuous bounded above function $G: X \rightarrow \mathbb{R}$ and an arbitrary fixed integer $k \geq 0$ such that

$$
\rho[x, T x] \leq G(T x)-G(x)+\cdots+G\left(T^{2 k+1} x\right)-G\left(T^{2 k} x\right)
$$

and $G\left(T^{2 i} x\right) \leq G\left(T^{2 i+1} x\right)$ for $i=0,1, \ldots, k$ and for every $x \in X$. Then $T$ has a fixed point in $X$.

As a further application of the preceding ideas we have the following geometric statement on upper transversal spaces. 
Theorem 10. Let $T$ be a self-map on an orbitally CS-complete upper transversal space $(X, \rho)$. Suppose that there exists an upper semicontinuous bounded above function $G: X \rightarrow[a,+\infty)$ for some $a>0$ such that

$$
\rho[x, T x] \leq \frac{1}{G(x)}-\frac{1}{G(T x)}
$$

for every $x \in X$. Then $T$ has a fixed point in $X$.

The proof of this statement is an analogous to the proof of the Theorem 8 based on Axiom of Choice.

As a directly extension of Theorem 10, we obtain the following result of fixed point on orbitally CS-complete upper transversal spaces.

Theorem 11. Let $T$ be a self-map on an orbitally CS-complete upper transversal space $(X, \rho)$. Suppose that there exists an upper semicontinuous bounded above function $G: X \rightarrow[a,+\infty)$ for some $a>0$ such that

$$
\rho[x, T x] \leq \sum_{i=0}^{\infty}\left(\frac{1}{G\left(T^{2 i} x\right)}-\frac{1}{G\left(T^{2 i+1} x\right)}\right)
$$

and $G\left(T^{2 i} x\right) \leq G\left(T^{2 i+1} x\right)$ for $i \in \mathbb{N} \cup\{0\}$ and for every $x \in X$. Then $T$ has a fixed point in $X$. 10 .

The proof of this statement is a totally analogous with the proof of Theorem

As an immediate consequence of the preceding result we obtain the following statement of fixed point on upper transversal spaces.

Corollary 2. Let $T$ be a self-map on an orbitally CS-complete upper transversal space $(X, \rho)$. Suppose that there exist an upper semicontinuous bounded above function $G: X \rightarrow[a,+\infty)$ for some $a>0$ and an arbitrary fixed integer $k \geq 0$ such that

$$
\rho[x, T x] \leq \frac{1}{G(x)}-\frac{1}{G(T x)}+\cdots+\frac{1}{G\left(T^{2 k} x\right)}-\frac{1}{G\left(T^{2 k+1} x\right)}
$$

and $G\left(T^{2 i} x\right) \leq G\left(T^{2 i+1} x\right)$ for $i=0,1, \ldots, k$ and for every $x \in X$. Then $T$ has a fixed point in $X$.

We notice that for $k=0$ in Corollary 2, directly, we obtain Theorem 10. On the other hand, Corollary 2 is a consequence or Theorem 11, because inequality (3) widening of inequality (4).

In connection with the preceding results on upper transversal spaces hold the following general statements.

Theorem 12. Let $T$ be a self-map on an orbitally CS-complete upper transversal space $(X, \rho)$. Suppose that there exists a lower semicontinuous function $G: X \rightarrow$ $\mathbb{R}_{+}^{0}$ such that

$$
\rho[x, T x] \leq \sum_{i=0}^{\infty}\left(G\left(T^{2 i} x\right)-G\left(T^{2 i+1} x\right)\right)
$$


and $G\left(T^{2 i+1} x\right) \leq G\left(T^{2 i} x\right)$ for $i \in \mathbb{N} \cup\{0\}$ and for every $x \in X$. Then $T$ has a fixed point in $X$.

A brief suitable proof of this statement without Zorn's lemma may be found in Tasković [13].

Corollary 3. Let $T$ be a self-map on an orbitally CS-complete upper transversal space $(X, \rho)$. Suppose that there exists a lower semicontinuous function $G: X \rightarrow$ $\mathbb{R}_{+}^{0}$ such that

$$
\rho[x, T x] \leq G(x)-G(T x)
$$

for every $x \in X$, then $T$ has a fixed point in $X$.

This result (Corollary 3) is a directly extension the statement which are given Caristi [1] and Kirk-Caristi [2] with metric spaces on upper transversal spaces. For further facts for this see: Tasković [17].

Corollary 4. Let $T$ be a self-map on an orbitally CS-complete upper transversal space $(X, \rho)$ Suppose that there exist a lower semicontinuous function $G: X \rightarrow \mathbb{R}_{+}^{0}$ and an arbitrary fixed integer $k \geq 0$ such that

$$
\rho[x, T x] \leq G(x)-G(T x)+\cdots+G\left(T^{2 k} x\right)-G\left(T^{2 k+1} x\right)
$$

and $G\left(T^{2 i+1} x\right) \leq G\left(T^{2 i} x\right)$ for $i=0,1, \ldots, k$ and for every $x \in X$. Then $T$ has $a$ fixed point in $X$.

A first brief suitable proof of this statement based on Zorn's lemma may be found in: Tasković [15].

In connection with the preceding statements, we now show that the following facts hold.

Let $X$ be a topological space (or only a nonempty set), let $T: X \rightarrow X$ and let $A: X \times X \rightarrow \mathbb{R}_{+}^{0}$ be a given mapping. We shall introduce the concept of CA-variation iff: there exists a function $A: X \times X \rightarrow \mathbb{R}_{+}^{0}$ such that

$$
\sum_{n=0}^{\infty} A\left(T^{n} x, T^{n+1} x\right)<+\infty
$$

for arbitrary $x \in X$. In this case, if (5) holds, we say and that $T: X \rightarrow X$ is bounded variation or bounded A-variation.

Lemma 6. Let $X$ be a nonempty set, $T: X \rightarrow X$, and let $A: X \times X \rightarrow \mathbb{R}_{+}^{0}$ be $a$ given mapping. Then the following facts are mutually equivalent:

(a) $T$ is a bounded variation mapping on a nonempty set $X$ in the sense of the mapping $A$.

(b) There is a function $G: X \rightarrow \mathbb{R}_{+}^{0}$ such that holds the following inequality in the form

$$
A(x, T x) \leq G(x)-G(T x) \quad \text { for every } \quad x \in X .
$$

(c) There is a nonnegative sequence of real functions in the form $x \mapsto C_{n}(x, T x)$ such that the following inequality holds

$$
A\left(T^{n} x, T^{n+1} x\right) \leq C_{n}(x, T x)
$$


for every $n \in \mathbb{N} \cup\{0\}$ and for every $x \in X$, where $\sum_{n=0}^{\infty} C_{n}(x, T x)<+\infty$ for arbitrary $x \in X$.

A first brief proof of this result (Lemma 6), in 1991, may be found in: Tasković [9]. The proof is an analogous with the proof of Lemma 1.

As a further application of the preceding ideas we have the following geometric statement on upper transversal spaces.

Theorem 13. Let $T$ be a self-map on an orbitally CS-complete upper transversal space $(X, \rho)$. Supppse that there exists a lower semicontinuous function $G: X \rightarrow$ $[a,+\infty)$ for some $a>0$ such that

$$
\rho[x, T x] \leq \sum_{i=0}^{\infty}\left(\frac{1}{G\left(T^{2 i+1} x\right)}-\frac{1}{G\left(T^{2 i} x\right)}\right)
$$

and $G\left(T^{2 i+1} x\right) \leq G\left(T^{2 i} x\right)$ for $i \in \mathbb{N} \cup\{0\}$ and for every $x \in X$. Then $T$ has a fixed point in $X$.

A brief suitable proof of this statement without Zorn's lemma may be found in: Tasković [13].

As an immediate consequence of the preceding result we obtain the following statement of fixed point.

Corollary 5. Let $T$ be a self-map on an orbitally CS-complete upper transversal space $(X, \rho)$. Suppose that there exist a lower semicontinuous function $G: X \rightarrow$ $[a,+\infty)$ for some $a>0$ and an arbitrary fixed integer $k \geq 0$ such that

$$
\rho[x, T x] \leq \frac{1}{G(T x)}-\frac{1}{G(x)}+\cdots+\frac{1}{G\left(T^{2 k+1} x\right)}-\frac{1}{G\left(T^{2 k} x\right)}
$$

and $G\left(T^{2 i+1} x\right) \leq G\left(T^{2 i} x\right)$ for $i=0,1, \ldots, k$ and for every $x \in X$. Then $T$ has $a$ fixed point in $X$.

We notice that, Corollary 5 is a consequence of Theorem 13, because inequality (6) widening of inequality (7).

Annotation 7. For any nonempty set $Y$ in the upper transversal space $X$, the diameter of $Y$ is defined as

$$
\operatorname{diam}(Y):=\sup \{\rho[x, y]: x, y \in X\}
$$

where $A \subset B$ implies $\operatorname{diam}(A) \leq \operatorname{diam}(B)$. The relation $\operatorname{diam}(Y)=0$ holds if and only if $Y$ is a one point set.

Elements of an upper transversal space will usually be called points. Given an upper transversal space $(X, \rho)$, with the bisection function $\psi:\left(\mathbb{R}_{+}^{0}\right)^{2} \rightarrow \mathbb{R}_{+}^{0}$ and a point $z \in X$, the open ball of center $z$ and radius $r>0$ is the set

$$
\psi(B(z, r))=\{x \in X: \rho[z, x]<r\} .
$$

The convergence $x_{n} \rightarrow x$ as $n \rightarrow \infty$ in the upper transversal space $(X, \rho)$ means that the following fact holds that

$$
\rho\left[x_{n}, x\right] \rightarrow 0 \quad \text { as } n \rightarrow \infty,
$$

or equivalently, for every $\varepsilon>0$ there exist on integer $n_{0}$ such that the relation $n \geq n_{0}$ implies $\rho\left[x_{n}, x\right]<\varepsilon$. 
The sequence $\left\{x_{n}\right\}_{n \in \mathbb{N}}$ in the upper transversal space $(X, \rho)$ is called transversal sequence (or upper Cauchy sequence) iff: for every $\varepsilon>0$ there is an $n_{0}=n_{0}(\varepsilon)$ such that

$$
\rho\left[x_{n}, x_{m}\right]<\varepsilon \text { for all } n, m \geq n_{0} .
$$

Let $(X, \rho)$ be an upper transversal space and $T: X \rightarrow X$. We notice, from Tasković [16], that a sequence of iterates $\left\{T^{n}(x)\right\}_{n \in \mathbb{N}}$ in $X$ is said to be transversal sequence if and only if

$$
\lim _{n \rightarrow \infty}\left(\operatorname{diam}\left\{T^{k}(x): k \geq n\right\}\right)=0 .
$$

In this sense, an upper transversal space is called upper complete iff every transversal sequence converges. Also, a space $(X, \rho)$ is said to be upper orbitally complete (or upper $T$-orbitally complete) iff every transversal sequence which contained in $\mathcal{O}(x)$ for some $x \in X$ converges in $X$.

A function $f$ mapping $X$ into reals is T-orbitally lower semicontinuous at $p \in X$ iff $\left\{x_{n}\right\}_{n \in \mathbb{N}}$ is a sequence in $\mathcal{O}(x)$ and $x_{n} \rightarrow p(n \rightarrow \infty)$ implies that $f(p) \leq \lim$.inf $f\left(x_{n}\right)$.

We are now in a position to formulate the following statement, which is roofing for a great number of known result on metric spaces in the fixed point theory.

Theorem 14. Let $T$ be a mapping of an upper transversal space $(X, \rho)$ into itself and let $X$ be upper T-orbitally complete. Suppose that there exists a function $\varphi: \mathbb{R}_{+}^{0} \rightarrow \mathbb{R}_{+}^{0}$ satisfying

$$
\left(\forall t \in \mathbb{R}_{+}\right)\left(\varphi(t)<t \text { and } \limsup _{z \rightarrow t+0} \varphi(z)<t\right)
$$

such that

$$
\rho[T x, T y] \leq \varphi\left(\operatorname{diam}\left\{x, y, T x, T y, T^{2} x, T^{2} y, \ldots\right\}\right)
$$

for all $x, y \in X$. If $x \mapsto \operatorname{diam} \mathcal{O}(x)$ or $x \mapsto \rho[x, T x]$ is T-orbitally lower semicontinuous, then $T$ has a unique fixed point $\zeta \in X$ and $\left\{T^{n}(x)\right\}_{n \in \mathbb{N}}$ converges to $\zeta$ for every $x \in X$.

A variant brief proof of this statement may be found in: Tasković [14] with the application of the following context.

Lemma 7. (Tasković [14]). Let the mapping $\varphi: \mathbb{R}_{+}^{0} \rightarrow \mathbb{R}_{+}^{0}$ have the property (Iu). If the sequence $\left(x_{n}\right)$ of nonegative real numbers satisfies the condition

$$
x_{n+1} \leq \varphi\left(x_{n}\right), \quad n \in \mathbb{N},
$$

then the sequence $\left(x_{n}\right)$ tends to zero. The velocity of this convergence is not necessarily geometrical.

A brief proof of this fact may be found in: Tasković [14]. A brief second proof of this statement may be found in: Tasković [17].

As immediate consequences of the preceding Theorem 14 we obtain directly the following interesting cases of $(\mathrm{J})$ :

(a) There exists a nondecreasing function $\varphi: \mathbb{R}_{+}^{0} \rightarrow \mathbb{R}_{+}^{0}$ satisfying the following fact that $\lim \sup _{z \rightarrow t+0} \varphi(z)<t$ for every $t \in \mathbb{R}_{+}$such that

$$
\rho[T x, T y] \leq \varphi(\operatorname{diam}\{x, y, T x, T y\})
$$

for all $x, y \in X$.

(b) (Special linear case of $(\mathrm{J})$ for $\varphi(t)=\alpha t$ ). There exists a constant $\alpha \in[0,1)$ such that for all $x, y \in X$ the following inequality holds

$$
\rho[T x, T y] \leq \alpha \operatorname{diam}\{x, y, T x, T y\}
$$

i.e., equivalently to

$$
\rho[T x, T y] \leq \alpha \max \{\rho[x, y], \rho[x, T x], \rho[y, T y], \rho[x, T y], \rho[y, T x]\} .
$$


(c) There exists a nondecreasing function $\varphi: \mathbb{R}_{+}^{0} \rightarrow \mathbb{R}_{+}^{0}$ satisfying the following fact that $\lim \sup _{z \rightarrow t+0} \varphi(z)<t$ for every $t \in \mathbb{R}_{+}$such that

$$
\rho[T x, T y] \leq \varphi\left(\operatorname{diam}\left\{x, y, T x, T y, \ldots, T^{k} x, T^{k} y\right\}\right)
$$

for an arbitrary fixed integer $k \geq 0$ and for all $x, y \in X$.

(d) There exists an increasing mapping $f:\left(\mathbb{R}_{+}^{0}\right)^{5} \rightarrow \mathbb{R}_{+}^{0}$ satisfying the following fact that $\lim \sup _{z \rightarrow t+0} f(z, z, z, z, z)<t$ for every $t \in \mathbb{R}_{+}$such that for all $x, y \in X$ is

$$
\rho[T x, T y] \leq f(\rho[x, y], \rho[x, T x], \rho[y, T y], \rho[x, T y], \rho[y, T x]) .
$$

Annotation 8. Let $X$ be a topological space, let $T: X \rightarrow X$, and let $A: X \times X \rightarrow \mathbb{R}_{+}^{0}$ be a given mapping. We shall introduce the concept of CS-convergence in a space $X$; i.e., a topological space $X$ satisfies the condition of CS-convergence iff: $\left\{x_{n}\right\}_{n \in \mathbb{N}}$ is an arbitrary sequence in $X$ and $\sum_{i=1}^{\infty} A\left(x_{i}, x_{i+1}\right)<+\infty$ implies that $\left\{x_{n}\right\}_{n \in \mathbb{N}}$ has a convergent subsequence in $X$.

On the other hand, a topological space $X$ satisfies the following condition of orbitally CS-convergence iff: $\left\{T^{n} x\right\}_{n \in \mathbb{N} \cup\{0\}}$ for $x \in X$ is an arbitrary iteration sequence in $X$ and $\sum_{n=0}^{\infty} A\left(T^{n} x, T^{n+1} x\right)<+\infty$ (for $\left.x \in X\right)$ implies that $\left\{T^{n} x\right\}_{n \in \mathbb{N} \cup\{0\}}$ has a convergent subsequence in $X$.

In connection with this, we notice that parallely with the statements of this section (Theorems 6 to 14 ) we have directly that holds and adequate statements for topological spaces. Naimely, Theorems 6 to 14 insists that holds if: 1) $X$ is an upper transversal space to change with $X$ is a topological space, and 2) the orbitally CS-convergence of $(X, \rho)$ to change with the orbitally CS-convergence of a topological space $X$ in the sense of the function $A: X \times X \rightarrow \mathbb{R}_{+}^{0}$ satisfying $A(a, b)=0$ iff $a=b$. For further facts of this see: Tasković [5], [14], and [17].

In connection with the preceding, we shall introduce the concept of upper topological space. In this sense, the function $A: X \times X \rightarrow \mathbb{R}_{+}^{0}$ is called an upper transverse on a nonempty set $X$ (or upper transversal) iff: $A(x, y)=0$ if and only if $x=y$ for all $x, y \in X$.

An upper topological space $(X, A)$ is a topological space $X$ together with a given upper transverse $A$ on $X$.

Otherwise, the function $A$ is called a semiupper transverse on a nonempty set $X$ iff: $A(x, y)=0$ implies $x=y$ for all $x, y \in X$. A semiupper topological space $(X, A)$ is a topological space $X$ together with a given semiupper transverse $A$ on $X$.

We notice, in connection with the preceding, that Theorems 6 to 14 de facto hold and for upper topological spaces in suitable context and transcription.

Mappings of the classical $A$-variation. Let $X$ be a topological space (or only a nonempty set), let $T: X \rightarrow X$ and let $A: X \times X \rightarrow \mathbb{R}_{+}^{0}$ be a given mapping. We shall introduce the concept of classical $A$-variation iff: there exists a function $A: X \times X \rightarrow \mathbb{R}_{+}^{0}$ such that

$$
\sum_{n=0}^{\infty} A\left(T^{n} x, T^{n} y\right)<+\infty
$$

for all $x, y \in X$. In this sense, if $(X, \rho)$ is an upper transversal space and if $A=\rho$, then we say that $T: X \rightarrow X$ is classical bounded variation (or classical $\rho$-variation).

Lemma 8. Let $X$ be a nonempty set, $T: X \rightarrow X$, and let $A: X \times X \rightarrow \mathbb{R}_{+}^{0}$ be a given mapping. Then the following facts are muttually equivalent:

(a) $T$ is mapping of classical A-variation. 
(b) There exists a function $G: X \times X \rightarrow \mathbb{R}_{+}^{0}$ such that holds the following inequality in the form

$$
A(x, y) \leq G(x, y)-G(T x, T y) \quad \text { for all } \quad x, y \in X .
$$

(c) There is a nonnegative sequences of real functions in the form $(x, y) \mapsto C_{n}(x, y)$ such that the following inequality holds

$$
A\left(T^{n} x, T^{n} y\right) \leq C_{n}(x, y) \quad \text { for all } x, y \in X,
$$

and for all $n \in \mathbb{N} \cup\{0\}$, where $\sum_{n=0}^{\infty} C_{n}(x, y)$ is a convergent series for all $x, y \in X$.

A brief suitable proof of this statement may be found in: Tasković [5]. On the other hand, from the preceding facts: (a), (b), and (c) of Lemma 8 are muttually equivalent also and with: There exist a function $G: X \times X \rightarrow \mathbb{R}_{+}^{0}$ and an arbitrary fixed integer $k \geq 0$ such that

$$
A(x, y) \leq G(x, y)-G(T x, T y)+\cdots+G\left(T^{2 k} x, T^{2 k} y\right)-G\left(T^{2 k+1} x, T^{2 k+1} y\right)
$$

and $G\left(T^{2 i+1} x, T^{2 i+1} y\right) \leq G\left(T^{2 i} x, T^{2 i} y\right)$ for $i=0,1, \ldots, k$ and for all $x, y \in X$. (The proof of this fact is similar with the proof of Lemma 8.)

In connection with the preceding, let $X$ topological space, let $T: X \rightarrow X$ and let $A: X \times X \rightarrow$ $\mathbb{R}_{+}^{0}$ be a function. A topological space $X$ satisfies the condition of classical TCS-convergence if for all $x, y \in X$ the condition $A\left(T^{n} x, T^{n} y\right) \rightarrow 0(n \rightarrow \infty)$ implies that $\left\{T^{n} x\right\}_{n \in \mathbb{N}}$ has a convergent subsequence in $X$.

Theorem 15. Let $T$ be a self-map on an upper topological space $(X, A)$ which is with the property of classical TCS-convergence. If $T$ is a classical A-variation mapping and if $x \mapsto A(x, T x)$ is a $T$-orbitally lower semicontinuous function, then $T$ has a unique fixed point in $X$.

A brief suitable proof of this statement with Zorn's lemma may be found in: Tasković [5]. Also, for a localization form of this statement see: Tasković [17].

As an immediate application of the preceding facts, as a directly consequence of former statements, we obtain the following result.

Theorem 16. Let $T$ be a self-map on an upper topological space $(X, A)$ which is with the property of classical TCS-convergence. Suppose that there a function $G: X \times X \rightarrow \mathbb{R}_{+}^{0}$ such that

$$
A(x, y) \leq \sum_{i=0}^{\infty}\left(G\left(T^{2 i} x, T^{2 i} y\right)-G\left(T^{2 i+1} x, T^{2 i+1} y\right)\right)
$$

and $G\left(T^{2 i+1} x, T^{2 i+1} y\right) \leq G\left(T^{2 i} x, T^{2 i} y\right)$ for $i \in \mathbb{N} \cup\{0\}$ and for all $x, y \in X$. If $x \mapsto A(x, T x)$ is a T-orbitally lower semicontinuous function, then $T$ has a unique fixed point in $X$.

A brief proof of this statement via Zorn's lemma may be found in: Tasković [5]. Also, for a localization form of this statement see: Tasković [13] and [17].

Convexity on upper transversal spaces. The set $C$ in linear space is convex (=upper convex) if for $x, y \in C$ and $\lambda \in[0,1]$ implies that we have $\lambda x+(1-\lambda) y \in C$. The upper transversal space $(X, \rho)$ is called upper convex (or transversal upper convex) if for any two different points $x, y \in X$ there is a point $z \in X(z \neq x, y)$ such that

$$
\rho[x, y]=\rho[x, z]+\rho[z, y] .
$$

In connection with this, if $C \subset X$ is a convex set of a transversal upper normed $\operatorname{space}(X,\|\cdot\|)$, then $C$ also and transversal upper convex space with $\rho[x, y]=\| x-$ $y \|$, because for any two different points $x, y \in C$ there is a point $z:=(x+y) / 2 \in C$ $(z \neq x, y)$ such that $(\mathrm{Cu})$ holds. For further facts on upper transversal normed spaces see: Tasković [17]. 
The following statements are very connection with the famous Schauder's problem for normed spaces and arbitrary linear topological spaces. For a solution of Schauder's problem see: Tasković [12].

Proposition 4a. Let $C$ be a nonempty convex compact subset of a linear topological space $X$ and suppose $T: C \rightarrow C$ is an upper continuous mapping. Then $T$ has a fixed point in $C$.

A proof of this statement we can give totally analogous with the proof of Proposition 4 .

Proposition 5a. Suppose that $C$ is a nonempty convex compact subset of $\mathbb{R}^{n}$, and that $T: C \rightarrow C$ is an upper continuous mapping. Then $T$ has a fixed point in $C$.

This statement is an analogous with the famous Brouwer's theorem. We can now formulate this statement in a manner valid for all transversal upper normed linear spaces.

Proposition 6a. Let $C$ be a nonempty, compact, convex subset of a transversal upper normed space $X$, and suppose $T: C \rightarrow C$ is an upper continuous operator. Then $T$ has a fixed point in $C$.

This statement is an analogous with the famous Schauder's theorem. We notice that Propositions 5a and 6a are directly consequences of Proposition 4a. For further facts on Schauder's problem see: Tasković [12] and [17].

A characterization of the class of upper contractive mappings. In this part of this paper we introduce the concept of upper transversal contraction mapping $T$ of a transversal upper space $X$, i.e., of a mapping $T: X \rightarrow X$ such that for all $x, y \in X$ there exists a sequence of nonnegative real functions $\mathcal{A}_{n, r}(x, y)$ with $\mathcal{A}_{n, r}(x, y) \rightarrow 0(r \geq n \rightarrow \infty)$ and positive integer $m(x, y)$ such that

$$
\rho\left[T^{n} x, T^{r} y\right] \leq \mathcal{A}_{n, r}(x, y) \quad \text { for all } \quad r>n \geq m(x, y) .
$$

Also, in this part we introduced the concept of upper $\sigma$-contraction $T$ of an upper transversal space $X$ into itself, i.e., of a mapping $T: X \rightarrow X$ such that for all $x, y \in X$ there exist numbers $C_{n}(x, y)>0$ and $K(x, y)>0$ such that

$$
\rho\left[T^{n} x, T^{n} y\right] \leq C_{n}(x, y) K(x, y), \quad \text { for } \quad n \in \mathbb{N},
$$

where $\sum_{n=1}^{\infty} C_{n}(x, y)$ is a convergent series for all $x, y \in X$.

We will in further denote by $\mathcal{D}\left(\mathbb{R}_{+}^{0}\right)$ the set of upper bisection functions $\psi$ : $\left(\mathbb{R}_{+}^{0}\right)^{2} \rightarrow \mathbb{R}_{+}^{0}$ which are incresing satisfying $\psi(t, t) \leq t$ for every $t \in \mathbb{R}_{+}^{0}$.

Proposition 10. Necessary and sufficient conditions that a mapping $T$ of an upper transversal space $(X, \rho)$ with a bisection function $\psi \in \mathcal{D}\left(\mathbb{R}_{+}^{0}\right)$ into itself and with continuity of $\rho$ has the following properties: (a) $T$ has a unique fixed point $\zeta \in X$, (b) $x_{n}=T^{n}(x) \rightarrow \zeta(n \rightarrow \infty)$ for every $x \in X$, and

$$
\rho\left[T^{n} x, \zeta\right] \leq \mathcal{A}_{n}(x, T x), \quad \text { for } n \geq m(x),
$$


where $x \mapsto \mathcal{A}_{n}(x, T x)$ are real nonnegative functions with $\mathcal{A}_{n}(x, T x) \rightarrow 0(n \rightarrow$ $\infty)$ are the following ones: (e) $X$ is upper $T$-orbitally complete, $(f) T$ is upper orbitally continuous, and $(g) T$ is upper transversal contraction mapping, where

$$
\mathcal{A}_{n, r}(x, y)=\max \left\{\mathcal{A}_{n}(x, T x), \mathcal{A}_{r}(y, T y)\right\} .
$$

The proof of this statement is very similar with the proof suitable for the metric spaces. For this see: Tasković [5] and [17].

Proposition 11. Let $(X, \rho)$ be an upper transversal space with continuity of $\rho$ and with a bisection function $\psi \in \mathcal{D}\left(\mathbb{R}_{+}^{0}\right)$ and $T$ a map of $X$ into itself. The conditions (a), (b) of proposition 10, and for each $x \in X$

$$
\rho\left[T^{n} x, \zeta\right] \leq\left(\sum_{k=n}^{\infty} C_{k}(x, T x)\right) K(x, T x), \quad \text { for } \quad n \in \mathbb{N},
$$

are necessary for the conditions $(f),(e)$ of proposition 10, and upper $\sigma$-contraction. On the other hand, the conditions (a), (b) of proposition 10, and ( $h$ ) with

$$
\sum_{n=1}^{\infty}\left(\sum_{k=n}^{\infty} C_{k}(x, T x)\right)<+\infty
$$

are sufficient for (f), (e) of proposition 10, and upper $\sigma$-contraction.

Now we shall show how Proposition 11 can be applied for investigation of solvability of an integral equation of the form (IE).

Proposition 12. Let $K:[\alpha, \beta] \times[\alpha, \beta] \times \mathbb{R} \rightarrow \mathbb{R}$ be continuous (=upper continuous) and let satisfy the Lipschitz condition of the form (Lip). Then for any $v \in C[\alpha, \beta]$ the equation (IE) has a unique solution $u \in C[\alpha, \beta]$. Moreover, defining a sequence of functions $\left\{u_{n}\right\}_{n \in \mathbb{N}}$ inductively by choosing any $u_{0} \in C[\alpha, \beta]$ and setting

$$
u_{n+1}(x)=v(x)+\lambda \int_{\alpha}^{x} K\left(x, t, u_{n}(t)\right) d t,
$$

the sequence $\left\{u_{n}\right\}_{n \in \mathbb{N}}$ converges (uniformly) on $[\alpha, \beta]$ to the unique solution $u$ of the equation (IE).

\section{REFERENCES}

[1] J. Caristi, Fixed point theorems for mappings satisfying inwardness conditions, Trans. Amer.Math. Soc., 215(1976), 241-251.

[2] W.A. Kirk and J. Caristi, Mapping theorems in metric and Banach spaces, Bull.Acad.Polon., 23(1975), 891-894.

[3] M. A. Krasnosleskij and V. Stečenko, $K$ teorii uravnenia $s$ vognutim operatorami, Sib.Math.J., 10(1969), 562-572.

[4] M. R. Tasković, A monotone principle of fixed points, Proc.Amer.Math.Soc., 94(1985), 427432.

[5] M. R. Tasković, Nonlinear Functional Analysis, Second Book: Monographs - Global Convex Analysis - General convexity, Variational methods and Optimization, Zavod za udžbenike i nastavna sredstva and Vojnoizdavački zavod, Beograd 2001, (in Serbian), 1223 pages. 
[6] M. R. Tasković, Transversal and fixed points, forks, general convex functions and apllications, Monographs: Zavod za udžbenike i nastavna sredstva and Vojnoizdavački zavod, Beograd 2001, Nonlinear Functional Analysis, Vol 2(2001), 1067-1172.

[7] M. R. Tasković, General expansion mappings on topological spaces, Scientiae Math. Japonicae, 54, No.1(2001), 61-67; e4, 497-503.

[8] M. R. Tasković, New geometric fixed point theorems, Math. Moravica 2(1998), 143-148.

[9] M. R. Tasković, Extensions of Brouwer's theorem, Math. Japonica, 36(1991), 685-693.

[10] M. R. Tasković, The axiom of choice, fixed point theorems, and inductive ordered sets, Proc.Amer.Math.Soc., 116(1992), 897-904.

[11] M. R. Tasković, Schauder's 54th problem in Scottish book, Mathematica Moravica 2(1998), 121-132.

[12] M. R. Tasković, On Schauder's 54th problem in Scottish book revisited, Math. Moravica, 6(2002), 119-126.

[13] M. R. Tasković, Extension of theorems by Krasnoselskij, Stečenko, Dugundji, Granas, Kiventidis, Romaguera, Caristi and Kirk, Math. Moravica, 6(2002), 109-118.

[14] M. R. Tasković, Nonlinear Functional Analysis, Fundamental elements of theory, First Book. Monographs, Zavod za udžbenike i nastavna sredstva, Beograd 1993, 792 p.p. (SerboCroation). English summary: Comments only new main results of this book. Vol. 1(1993), 713-752.

[15] M. R. Tasković, A directly extension of Caristi fixed point theorem, Math. Moravica, 1(1997), 105-108.

[16] M. R. Tasković, Transversal spaces, Mathematica Moravica, 2(1998), 133-142.

[17] M. R. Tasković, Theory of transversal point, spaces and forks, Monographs of a new theory, Beograd 2003, (in Serbian), 1000 pages, to appear.

MatematiČKi FAKULtet

11000 Beograd, P.O. Box 550

Serbia \& Montenegro

E-mail address: tassneza@eunet.yu 